\title{
STRONG DIFFERENTIABILITY OF LIPSCHITZ FUNCTIONS
}

\author{
BY
}

\section{J. NEUGEBAUER}

\begin{abstract}
Let $F$ be a differentiation basis in $R^{n}$, i.e., a family of measurable sets $S$ contracting to 0 such that $\left\|M_{F} f\right\|_{p}<A_{p}\|f\|_{p}$, where $M_{F}$ is the Hardy-Littlewood maximal operator. For $f \in \Lambda_{\alpha}^{p q}$, we let $E_{F}(f)$ be the complement of the Lebesgue set of $f$ relative to $F$, and we show that $E_{F}$ has $L_{\alpha}^{p q}$-capacity 0 , where $L_{\alpha}^{p q}$ is a capacity associated with $\Lambda_{\alpha}^{p q}$ in much the same way as the Bessel capacity $B_{\alpha p}$ is associated with $L_{\alpha}^{p}$.
\end{abstract}

1. With the Bessel potential space $L_{\alpha}^{p}\left(R^{n}\right)$ there is associated the Bessel capacity $B_{\alpha p}$ which is defined for $E \subset R^{n}$ by

$$
B_{\alpha p}(E)=\inf \left\{\|g\|_{p}^{p}: g \in L^{p}, g \geqslant 0, G_{\alpha} * g \geqslant 1 \text { on } E\right\},
$$

where $G_{\alpha}$ is the Bessel kernel given by $\widehat{G_{\alpha}}(x)=\left(1+4 \pi^{2}|x|^{2}\right)^{-\alpha / 2}[5$, p. 132]. The capacity $B_{\alpha p}$ is an outer measure on $R^{n}$ and its relation to $H^{r}$, Hausdorff measure of dimension $r$, is given by the following [4]. If $p>1, \alpha p<n$, then $H^{n-\alpha p}(E)=0$ implies $B_{\alpha p}(E)=0$, and $B_{\alpha p}(E)=0$ implies $H^{n-\alpha p+\varepsilon}(E)=0$ for every $\varepsilon>0$.

Let $F$ be a family of measurable sets $S \subset R^{n}$ with $0<|S|<\infty$, and let $S \rightarrow 0$ stand for the generic notation for the limit as $j \rightarrow \infty$ of any sequence $\left\{S_{j}\right\} \subset F$ such that, for any $\varepsilon>0, S_{j} \subset\{|x|<\varepsilon\}, j \geqslant j(\varepsilon)$. With such a family we associate the Hardy-Littlewood maximal operator

$$
M_{F} f(x)=\sup \left\{\frac{1}{|S|} \int_{S+x}|f(y)| d y: S \in F\right\} .
$$

The behavior of $M_{F} f$ is decisive in the study of the differentiability of the integral. If, for example, $\left\|M_{F} f\right\|_{p} \leqslant A_{p}\|f\|_{p}$ (there are many interesting families $F$ with this property [3]), then the set

$$
E_{F}(f)=\left\{x: \frac{1}{|S|} \int_{S+x}|f(y)-f(x)| d y \nrightarrow 0 \text { as } S \rightarrow 0\right\}
$$

has measure 0 for $f \in L^{p}$. If $f \in L_{\alpha}^{p}$, then as shown in [2] the exceptional set $E_{F}(f)$ has even $B_{a p}$-capacity 0 .

The purpose of this paper is to prove an analogous result for the Lipschitz spaces $\Lambda_{\alpha}^{p q}\left(R^{n}\right)$ (see [5], [6]), using instead of $B_{\alpha p}$ a "Lipschitz" capacity $L_{\alpha}^{p q}$.

Received by the editors February 20, 1976.

AMS (MOS) subject classifications (1970). Primary 26A54, 26A16; Secondary 46E35.

Key words and phrases. Lipschitz spaces, Lipschitz capacity, differentiation.

- American Mathematical Society 1978 
In view of the inclusion relations

$$
L_{\alpha}^{p} \subset \Lambda_{\alpha}^{p r} \subset \Lambda_{\alpha}^{p q} \subset L_{B}^{p}, \quad p>1,
$$

$r=\max (p, 2), q \geqslant r, \beta<\alpha[6$, pp. 441, 452] our differentiability result lies then intermediate to those of [2].

We recall that, if $F$ is the family of all oriented rectangles, a nonregular family, then $\left\|M_{F} f\right\|_{p}<A_{p}\|f\|_{p}, 1<p<\infty$ [5]. For regular families $F$, i.e., those for which there is $c>0$ such that every $E \in F$ lies in a sphere $S$ with $|S|<c|E|$, the size of $E_{F}(f)$ for $f \in \Lambda_{\alpha}^{p q}$ can be studied with the techniques developed in [8], especially the mean value property $\int_{|y|<r} G_{\alpha}(x-y) d y<$ $c G_{\alpha}(x) r^{n}$. One sees easily that this tool is no longer available for nonregular families, and its substitute $\left\|M_{F} f\right\|_{p} \leqslant A_{p}\|f\|_{p}$, which is not a condition on the kernel $G_{\alpha}$, requires one to study the interplay between $\Lambda_{\alpha}^{p q}$ and $M_{F}$.

2. Let $F$ be a family as in $\S 1$, and let $t F=\{t S: S \in F\}, 0<t \leqslant 1$, where $t S=\{t s: s \in S\}$. Let

$$
M_{\iota} f(x)=\sup \left\{\frac{1}{|t S|} \int_{t S+x}|f(y)| d y: S \in F\right\} .
$$
1.

LEMMA 1. If $\left\|M_{F} f\right\|_{p} \leqslant A_{p}\|f\|_{p}, f \in L^{p}$, then $\left\|M_{\imath} f\right\|_{p} \leqslant A_{p}\|f\|_{p}, 0<t \leqslant$

PROOF. $|t S|^{-1} \int_{t} S+x|f(u)| d u=|s|^{-1} \int_{S+x / t}|f(t v)| d v \leqslant M_{F}\left(\delta_{t} f\right)(x / t)$, where $\left(\delta_{t} f\right)(x)=f(t x)$. Hence

$$
\begin{aligned}
\left\|M_{t} f\right\|_{p} & <\left\{\int_{R^{n}} M_{F}\left(\delta_{L} f\right)\left(\frac{x}{t}\right)^{p} d x\right\}^{1 / p} \\
& =\left\{t^{n} \int_{R^{n}} M_{F}\left(\delta_{L} f\right)(u)^{p} d u\right\}^{1 / p}<t^{n / p} A_{p}\left\{\int_{R^{n}}\left|\left(\delta_{\nu} f\right)(x)\right|^{p} d x\right\}^{1 / p} \\
& =A_{p}\|f\|_{p^{.}}
\end{aligned}
$$

We note that $A_{p}$ is independent of $t$.

For $0<\alpha<1$, the Lipschitz space $\Lambda_{\alpha}^{p q}\left(R^{n}\right)$ consists of all functions $f \in L^{p}\left(R^{n}\right)$ for which the norm

$$
\|f\|_{p}+\left\{\int_{R^{n}} \frac{\|f(x+t)+f(x-t)-2 f(x)\|_{p}^{q}}{|t|^{n+\alpha q}} d t\right\}^{1 / q}=\|f\|_{\Lambda_{a}^{\rho Q}}
$$

is finite [5, p. 151]; here $1 \leqslant p, q \leqslant \infty$. If $0<\alpha<1$, the above norm is equivalent to

$$
\|f\|_{p}+\left\{\int_{R^{n}} \frac{\|f(x+t)-f(x)\|_{p}^{q}}{|t|^{n+\alpha q}} d t\right\}^{1 / q}
$$


[5, p. 153]. If $\alpha>1, \Lambda_{\alpha}^{p q}$ is the collection of $f \in L^{p}\left(R^{n}\right)$ for which the norm $\|f\|_{p}+\sum_{j=1}^{n}\left\|\partial f / \partial x_{j}\right\|_{\Lambda_{a-1}^{a q}}<\infty\left[5\right.$, p. 153], where $\partial f / \partial x_{j}$ is taken in the sense of distribution.

LeMMa 2. Let $0<\alpha<1$ and $\left\|M_{F} f\right\|_{p}<A_{p}\|f\|_{p}, f \in L^{p}$. Then $\left\|M_{\downarrow} f\right\|_{\Lambda_{a}^{e q}}<$ $A_{p}\|f\|_{\Lambda_{a}^{\alpha q}}$.

Proof. By Lemma 1 we only need to show that $\left\|M_{F} f\right\|_{\Lambda_{a q}^{\rho a}}<A_{p}\|f\|_{\Lambda_{a}^{\rho a q}}$. If $f_{t}(x)=f(x+t)$, one easily verifies that $\left|M_{F} f(x+t)-M_{F} f(x)\right|<M_{F}\left(f_{t}-\right.$ $f)(x)$, and the result follows from (ii).

3. If $G_{\alpha}$ is the Bessel potential of order $\alpha>0$, and if $J_{\alpha} f=G_{\alpha} * f$, then $J_{\alpha}$ :

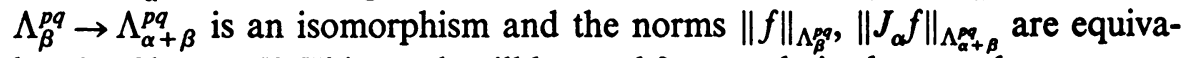
lent [5, Chapter 5]. This result will be used frequently in the sequel.

For $0<\alpha<\infty, 1<p, q \leqslant \infty$, we define, for $E \subset R^{n}$,

$$
L_{\alpha}^{p q}(E)=\inf \left\{\|g\|_{\Lambda_{\alpha}^{p q}: g}: 0 \text { and } g \geqslant 1 \text { on } E\right\} \text {. }
$$

For $0<\gamma<\alpha$ we define, for $E \subset R^{n}$,

$$
B_{\gamma}^{p q}(E)=\inf \left\{\|g\|_{\Lambda_{\gamma}^{p q}:} g \geqslant 0 \text { and } G_{\alpha-\gamma} * g>1 \text { on } E\right\} .
$$

It is easily verified that $L_{\alpha}^{p q}, B_{\gamma}^{p q}$ are capacities, i.e., they are monotone, countably subadditive, and assign 0 to $E=\varnothing$ (see [4, p. 251]).

The usefulness of $B_{\gamma}^{p q}$ is exhibited in the following lemma.

LeMma 3. $L_{\alpha}^{p q}(E)=0$ if and only if there exists $0<\gamma<\alpha$ such that $B_{\gamma}^{p q}(E)=0$.

Proof. $(\rightarrow)$ Let $0<\gamma<\alpha$ and let $\varepsilon>0$. Choose $g \in \Lambda_{\alpha}^{p q}$ so that $g>0$, $g>1$ on $E$, and $\|g\|_{\Lambda_{\alpha}^{\rho q}} \leqslant \varepsilon$. If $g=G_{\alpha-\gamma} * \psi, \psi \in \Lambda_{\gamma}^{p q}$, then \|\|$\psi \|_{\Lambda_{\gamma}^{\rho q}}<K \varepsilon$, and $B_{\gamma}^{p q}(E)=0$.

$(\leftarrow)$ If $B_{\gamma}^{p q}(E)=0$, then there is $g \geqslant 0$ in $\Lambda_{\gamma}^{p q}$ with $G_{\alpha-\gamma} * g>1$ on $E$ and $\|g\|_{\Lambda_{\gamma}^{\rho q}}<\varepsilon$. As before $\left\|G_{\alpha-\gamma} * g\right\|_{\Lambda_{\alpha}^{p q}}<K \varepsilon$, and $L_{\alpha}^{p q}(E)=0$.

LEMMA 4. Let $1<p<\infty$. The relation between the Bessel capacity $B_{\alpha p}$ and the Lipschitz capacity $L_{\alpha}^{p q}$ is given by

(i) $B_{\alpha p}(E)=0$ implies $L_{\alpha}^{p q}(E)=0 q>\max (p, 2)$.

(ii) $L_{\alpha}^{p q}(E)=0$ implies $B_{\gamma p}(E)=0,0<\gamma<\alpha$.

Proof. (i) We use Lemma 3 and verify that $B_{\gamma}^{p q}(E)=0$ for $0<\gamma<\alpha$. Since $B_{\alpha p}(E)=0$, there is $g \geqslant 0$ in $L^{p}$ such that $\|g\|_{p}<\varepsilon$ and $G_{\alpha} * g(x)>$ 1, $x \in E$. If $\psi=G_{\gamma} * g$, then $\|\psi\|_{\Lambda_{\gamma}^{p}}<M\|g\|_{p}<M \cdot \varepsilon$ [6, p. 452].

(ii) If $0<\gamma<\alpha$, we have $B_{\alpha-\gamma}^{p q}(E)=0$, and hence there is $g \geqslant 0$ with $\|g\|_{\Lambda_{a-\gamma}^{p q}}<\varepsilon$ and $G_{\gamma} * g(x) \geqslant 1, x \in E$. Since $\|g\|_{p}<\varepsilon$, we get $B_{\gamma p}(E)=0$.

Corollary. If $L_{\alpha}^{p q}(E)=0$ and $\alpha p>1$, then $H^{n-1}(E)=0$.

Proof. Let $0<\gamma<\alpha$ with $\gamma p>1$. Since $B_{\gamma p}(E)=0$, we see from [4, Theorem 22] that $H^{n-1}(E)=0$. 
The next lemma shows that $f \in \Lambda_{\alpha}^{p q}$ can be defined absolutely modulo sets of $L_{\alpha}^{p q}$-capacity 0 .

LeMma 5. Let $f \in \Lambda_{\alpha}^{p q}, 0<\gamma<\alpha$, and $f=G_{\alpha-\gamma} * \psi, \psi \in \Lambda_{\gamma}^{p q}$. Then $G_{\alpha-\gamma} *|\psi|(x)<\infty$ for $L_{\alpha}^{p q}$-a.e. $x$.

Proof. Let $E=\left\{x: G_{\alpha-\gamma} *|\psi|(x)=\infty\right\}$. Then

$$
\begin{aligned}
B_{\gamma}^{p q}(E) & <B_{\gamma}^{p q}\left\{x: G_{\alpha-\gamma} *|\psi|(x)>k\right\} \\
& =B_{\gamma}^{p q}\left\{x: G_{\alpha-\gamma} * \frac{|\psi|}{k}(x)>1\right\}<\frac{1}{k}\|\psi\|_{\Lambda_{\gamma}^{\rho q}} \rightarrow 0 \text { as } k \rightarrow \infty .
\end{aligned}
$$

The result now follows from Lemma 3.

LEMMA 6. Let $\psi_{j} \rightarrow f\left(\Lambda_{\alpha}^{p q}\right)$. Then there exists a subsequence $\left\{j_{i}\right\}$ such that $\psi_{j_{i}} \rightarrow$ f for $L_{\alpha}^{p q}$-a.e. $x$.

Proof. The proof is standard and we give it for the sake of completeness. Let $0<\gamma<\alpha, f=G_{\alpha-\gamma} * g, \psi_{j}=G_{\alpha-\gamma} * \phi_{j}, g, \phi_{j} \in \Lambda_{\gamma}^{p q}$. Then

$$
\begin{aligned}
B_{\gamma}^{p q}\left\{x:\left|\psi_{j}-f\right|(x)>\varepsilon\right\} & <B_{\gamma}^{p q}\left\{x: G_{\alpha-\gamma} *\left(\left|\phi_{j}-g\right| / \varepsilon\right)(x)>1\right\} \\
& <\left\|\phi_{j}-g\right\|_{\Lambda_{\gamma}^{p q}} / \varepsilon \rightarrow 0 \text { as } j \rightarrow \infty .
\end{aligned}
$$

If we select now $\left\{j_{i}\right\}$ such that for $A_{i}=\left\{x:\left|\psi_{j_{1}}-f\right|(x) \geqslant 1 / 2^{i}\right\}$ we have $B_{\gamma}^{p q}\left(A_{i}\right)<1 / 2^{i}$, then on $A=\cap_{k>1} \cup_{i>k} A_{i}, \psi_{j_{i}} \rightarrow f$ and $B_{\gamma}^{p q}(A)=0$.

LEMMA 7. If $\left\|M_{F} f\right\|_{p}<A_{p}\|f\|_{p}$ and $0<\alpha<1$, then $\left\|\int_{0}^{1} M_{t} g(x) d t\right\|_{\Lambda_{q}^{\infty}}<$ $A_{p}\|g\|_{\Lambda_{a}^{a}}$ where $M_{F}$ and $M_{t}$ are the maximal operators associated with $F$ and $t F$.

Proof. Let $\psi(x)=\int_{0}^{1} M_{t} g(x) d t$. Then $\|\psi\|_{p}<A_{p}\|g\|_{p}$ (Lemma 1), and

$$
\begin{aligned}
\|\psi(x+\tau)-\psi(x)\|_{p} & <\int_{0}^{1}\left\|M_{t}\left(g_{\tau}-g\right)\right\|_{p} d t \\
& <A_{p}\|g(x+\tau)-g(x)\|_{p},
\end{aligned}
$$

where $g_{\tau}(x)=g(x+\tau)$.

From Lemma 5 we deduce that for $g \in \Lambda_{\alpha}^{p q}, M_{t} g(x) \in L^{1}(d t,[0,1]$ for $L_{\alpha}^{p q}=$ a.e. $x$.

4. We are now ready to state and prove our differentiability results. We let $F$ be a family of sets as in $\S 1$, and we let $t F=\{t S: S \in F\}, 0<t<1$.

THEOREM 1. Assume that for $f \in L^{p}\left(R^{n}\right),\left\|M_{F} f\right\|_{p}<A_{p}\|f\|_{p}$. For $f \in$ $\Lambda_{\alpha}^{p q}\left(R^{n}\right)$, let 


$$
E_{t}(f)=\left\{x: \limsup _{S \rightarrow 0} \frac{1}{|t S|} \int_{t S+x}|f(y)-f(x)| d y>0\right\} .
$$

Then $L_{\alpha}^{p q}\left(E_{t}\right)=0$.

Proof. We have to verify that, for $\sigma>0, L_{\alpha}^{p q}\left(E_{\sigma}\right)=0$, where

$$
E_{\sigma}=\left\{x \in E_{t}: \limsup _{S \rightarrow 0} \frac{1}{|t S|} \int_{t S+x}|f(y)-f(x)| d y>\sigma\right\} .
$$

Let $0<\beta<\min (1, \alpha)$. By Lemma 3 we need to show that $B_{\beta}^{p q}(E)=0$. Let $\eta>0$ be given.

Let $C_{0}^{\infty}\left(R^{n}\right)$ be the space of infinitely differentiable functions with compact support. By [6, p. 444] there is a sequence $\left\{\psi_{j}\right\} \subset C_{0}^{\infty}$ such that $\psi_{j} \rightarrow f\left(\Lambda_{\alpha}^{p q}\right)$. Choose now $\psi_{j}^{\beta}, f^{\beta} \in \Lambda_{\beta}^{p q}$ with $G_{\alpha-\beta} * \psi_{j}^{\beta}=\psi_{j}$ and $G_{\alpha-\beta} * f^{\beta}=f$. Then $\psi_{j}^{\beta} \rightarrow f^{\beta}\left(\Lambda_{\beta}^{p q}\right)$, and from this we get that

$$
B_{\beta}^{p q}\{x:|\psi-f|(x)>\delta\}<\left\|\psi^{\beta}-f^{\beta}\right\|_{\Lambda_{\beta}^{q q}} / \delta=o(1) \text { as } j \rightarrow \infty .
$$

If $\Sigma=t S$, we have

$$
\begin{aligned}
\frac{1}{|\Sigma|} \int_{\Sigma+x}|f(y)-f(x)| d y< & \frac{1}{|\Sigma|} \int_{\Sigma+x}\left|f(y)-\psi_{j}(y)\right| d y \\
& +\frac{1}{|\Sigma|} \int_{\Sigma+x}\left|\psi_{j}(y)-\psi_{j}(x)\right| d y \\
& +\left|\psi_{j}(x)-f(x)\right| \\
& =A_{i j}(x)+A_{2 j}(x)+A_{3 j}(x) .
\end{aligned}
$$

(i) Since $\left\{x: M_{t}\left(f-\psi_{j}\right)(x) \geqslant \sigma / 3\right\} \subset\left\{x: G_{\alpha-\beta} * M_{t}\left(f^{\beta}-\psi_{j}^{\beta}\right)(x)>\right.$ $\sigma / 3\}$, we see that

$$
\begin{aligned}
B_{\beta}^{p q}\left\{x: M_{t}\left(f-\psi_{j}\right)(x)>\sigma / 3\right\} & \leqslant(3 / \sigma)\left\|M_{t}\left(f^{\beta}-\psi_{j}^{\beta}\right)\right\|_{\Lambda_{\beta}^{\beta}} \\
& <(3 / \sigma) A_{p}\left\|f^{\beta}-\psi_{j}^{\beta}\right\|_{\Lambda_{\beta}^{\rho \beta}}<\eta / 2, \text { if } j>j_{1}(\eta) .
\end{aligned}
$$

The next to the last inequality follows from Lemma 2 (applicable since $\beta<1)$. Since $A_{1 j}(x)<M_{t}\left(f-\psi_{j}\right)(x)$, there is a set $E_{1 j}$ such that $B_{\beta}^{p q}\left(E_{1 j}\right)<$ $\eta / 3, j \geqslant j_{1}(\eta)$, and $x \notin E_{1 j}$ implies $A_{1 j}(x)<\sigma / 3$.

(ii) Since $B_{\beta}^{p q}\left\{x: A_{3 j}(x) \geqslant \sigma / 3\right\}<(3 / \sigma)\left\|\psi_{j}^{\beta}-f^{\beta}\right\|_{\Lambda_{\beta}^{\rho q}}$, there is $j_{3}(\eta)$ such that for $j>j_{3}(\eta), A_{3 j}(x)<\sigma / 3$ except for $x \in E_{3 j}$ with $B_{\beta}^{p q}\left(E_{3 j}\right)<\eta / 2$.

(iii) Let now $j_{0} \geqslant \max \left[j_{1}(\eta), j_{3}(\eta)\right]$. Then there is $\tau(\sigma)$ such that $S \subset$ $B(0, \tau)$ implies $\left|\psi_{j_{0}}(y)-\psi_{j_{0}}(x)\right|<\sigma / 3, y \in t S+x$. If then $x \notin E_{1 j_{0}} \cup E_{3 j_{0}}$ and $S \subset B(0, \tau)$, we get $|\Sigma|^{-1} \int_{\Sigma+x}|f(y)-f(x)| d y \leqslant \sigma, \Sigma=t S$, and hence $E_{\sigma} \subset E_{1 j_{0}} \cup E_{3 j_{0}}$. Then $B_{\beta}^{p q}\left(E_{\sigma}\right)<\eta$, and the proof is complete.

THEOREM 2. Under the same hypothesis as for Theorem 1, we have for $L_{\alpha}^{p q}$-a.e. $x$, 
(i)

$$
\lim _{S \rightarrow 0} \frac{1}{|t S|} \int_{t S+x}|f(y)-f(x)| d y=0 \text {, a.e. } t, 0<t<1 \text {. }
$$

$$
\lim _{S \rightarrow 0} \int_{0}^{1} \frac{1}{|t S|} \int_{t S+x}|f(y)-f(x)| d y d t=0 .
$$

Proof. (i) Let

$$
E=\left\{(t, x): \limsup _{S \rightarrow 0} \frac{1}{|t S|} \int_{t S+x}|f(y)-f(x)| d y>0\right\},
$$

and let $E_{x}=\{t:(t, x) \in E\}$. We must show that $\left|E_{x}\right|=0$ for $L_{\alpha}^{p q}$-a.e. $x$.

Let $A_{\sigma}=\left\{x:\left|E_{x}\right|>\sigma\right\}$. Let $0<\beta<\min (1, \alpha)$ and choose $\left\{\psi_{j}\right\} \subset C_{0}^{\infty}$ so that $\psi_{j} \rightarrow f\left(\Lambda_{\alpha}^{p q}\right)$. By Lemma 6 we may assume that $\psi_{j}(x) \rightarrow f(x)$ for $L_{\alpha}^{p q}$-a.e. $x$. Let $E_{1}$ be the exceptional set, i.e., $L_{\alpha}^{p q}\left(E_{1}\right)=0$ and $x \notin E_{1}$ implies $\psi_{j}(x) \rightarrow f(x)$.

We need another exceptional set $E_{2}$. Since $M_{t}\left(f-\psi_{j}\right)(x)<G_{\alpha-\beta} * M_{t}\left(f^{\beta}\right.$ $\left.-\psi_{j}^{\beta}\right)(x)$ (notation as in the proof of Theorem 1 ), and $\psi_{j}^{\beta} \rightarrow f^{\beta}\left(\Lambda_{\beta}^{p q}\right)$, we see that

$$
\int_{0}^{1} M_{t}\left(f-\psi_{j}\right)(x) d t<G_{\alpha-\beta} * \int_{0}^{1} M_{t}\left(f^{\beta}-\psi_{j}^{\beta}\right)(x) d t \equiv \Psi_{j}(x) .
$$

By Lemma $7, \int_{0}^{1} M_{t}\left(f^{\beta}-\psi_{j}^{\beta}\right)(x) d t \in \Lambda_{\beta}^{p q}$, and hence $\Psi_{j} \in \Lambda_{\alpha}^{p q}$.

Next

$$
\left\{x: \int_{0}^{1} M_{t}\left(f-\psi_{j}\right)(x) d t>\varepsilon\right\} \subset\left\{x: G_{\alpha-\beta} * \frac{1}{\varepsilon} \int_{0}^{1} M_{t}\left(f^{\beta}-\psi_{j}^{\beta}\right)(x) d t>1\right\},
$$

and hence

$$
\begin{aligned}
B_{\beta}^{p q}\left\{x: \int_{0}^{1} M_{t}\left(f-\psi_{j}\right)(x) d t>\varepsilon\right\} & <\frac{1}{\varepsilon}\left\|\int_{0}^{1} M_{t}\left(f^{\beta}-\psi_{j}^{\beta}\right)(x) d t\right\|_{\Lambda_{\beta}^{\beta}} \\
& <\frac{A_{p}}{\varepsilon}\left\|f^{\beta}-\psi_{j}^{\beta}\right\|_{\Lambda_{\beta}^{\beta}}=0(1) \text { as } j \rightarrow \infty .
\end{aligned}
$$

We can now choose a subsequence $\left\{j_{i}\right\}$ so that $\int_{0}^{1} M_{t}\left(f-\psi_{j}\right)(x) d t \rightarrow 0$ for $L_{\alpha}^{p q}$-a.e. $x$ (see e.g. the proof of Lemma 6). We may assume that $\left\{j_{i}\right\}=\{j\}$. Let

$$
E_{2}=\left\{x: \int_{0}^{1} M_{t}\left(f-\psi_{j}\right)(x) d t \nrightarrow 0\right\} .
$$

Then $L_{\alpha}^{p q}\left(E_{2}\right)=0$.

We return now to the set $A_{\sigma}$ introduced at the beginning of the proof, and we claim that, for every $\sigma>0, A_{\sigma} \subset E_{1} \cup E_{2}$.

If we deny this, then there is $x_{0} \in A_{\sigma}$ and $x_{0} \notin E_{1} \cup E_{2}$. Then $\left|E_{x_{0}}\right|>\sigma$. If

$$
E_{x_{0} \lambda}=\left\{t: \limsup _{S \rightarrow 0} \frac{1}{|t S|} \int_{t S+x_{0}}\left|f(y)-f\left(x_{0}\right)\right| d y>\lambda\right\},
$$


then there is $\lambda>0$ with $\left|E_{x_{0} \lambda}\right|>\sigma$. We choose now $j_{0}$ so large that

(i) $\int_{0}^{1} M_{t}\left(f-\psi_{j_{0}}\right)\left(x_{0}\right) d t<\sigma \lambda / 3$,

(ii) $\left|\psi_{j_{0}}\left(x_{0}\right)-f\left(x_{0}\right)\right|<\lambda / 3$,

and we choose $\tau_{0}>0$ such that $S \subset B\left(0, \tau_{0}\right)$ implies

(iii) $\left|\psi_{j_{0}}(y)-\psi_{j_{0}}\left(x_{0}\right)\right|<\lambda / 3, y \in t S+x_{0}, 0<t<1$.

Since for $t \in E_{x_{0} \lambda}$,

$$
\lambda<M_{t}\left(f-\psi_{j_{0}}\right)\left(x_{0}\right)+\frac{2 \lambda}{3}, \quad S \subset B\left(0, \tau_{0}\right),
$$

we see that $E_{x_{0} \lambda} \subset\left\{t: M_{t}\left(f-\psi_{j}\right)\left(x_{0}\right)>\lambda / 3\right\}$. From this $\left|E_{x_{0} \lambda}\right|<$ $(3 / \lambda) \int_{0}^{1} M_{t}\left(f-\psi_{j_{0}}\right)\left(x_{0}\right) d t<\sigma$, a contradiction.

To prove (ii), we first observe that $\int_{0}^{1} M_{\downarrow} f^{\beta}(x) d t \in \Lambda_{\beta}^{p q}$ (Lemma 7), and hence $G_{\alpha-\beta} * \int_{0}^{1} M_{t} f^{\beta}(x) d t<\infty$ for $L_{\alpha}^{p q}$-a.e. $x$ (Lemma 5). Since $M_{\imath} f(x)<$ $G_{\alpha-\beta} * M_{\downarrow} f^{\beta}(x)$, we see that $M_{\downarrow} f(x) \in L^{1}\left(d t,[0,1]\right.$ for $L_{\alpha}^{p q}$-a.e. $x$. Finally,

$$
|f(x)|+M_{t} f(x)>\frac{1}{|t S|} \int_{t S+x}|f(y)-f(x)| d t \rightarrow 0 \text { as } S \rightarrow 0,
$$

and we only need to apply the Lebesgue dominated convergence theorem to obtain (ii).

COROLlary. Under the same hypothesis,

$$
\lim _{S \rightarrow 0} \int_{0}^{1} \cdots \int_{0}^{1} \frac{1}{\left|t_{1} t_{2} \cdots t_{k} S\right|} \int_{t_{1} t_{2} \cdots t_{k} S+x}|f(y)-f(x)| d y d t_{1} \cdots d t_{k}=0
$$

for $L_{\alpha}^{p q}$-a.e. $x$.

5. In this section we will study higher order differentiability. Let $F$ be a differentiation basis as in $\S 1$, and let $\delta(S)$ denote the diameter of $S$. We say that a function $f \in L^{p}\left(R^{n}\right)$ is in $t_{k}^{p}\left(x_{0}\right)$ with respect to $F$ if there exists a polynomial $\Pi_{x_{0}}(y)$ of degree $<k$ such that

$$
\left\{\frac{1}{|S|} \int_{S+x_{0}}\left|f(y)-\Pi_{x_{0}}(y)\right|^{p} d y\right\}^{1 / p}=o\left(\delta(S)^{k}\right), \text { as } S \rightarrow 0 .
$$

If $F$ is the family of all balls centered at the origin, this notion is due to Calderón and Zygmund [1], and for a general $F$ was introduced in [2].

THEOREM 3. Let $\left\|M_{F} f\right\|_{p}<A_{p}\|f\|_{p}$. If $f \in \Lambda_{\alpha}^{p q}\left(R^{n}\right)$, and $k$ is a nonnegative integer $<\alpha$, then $f \in t_{k}^{1}(x)$ with respect to $F$ for $L_{\alpha-k}^{p q}$-a.e. $x$.

Of course, the special case $k=0$ is Theorem 1. The proof of Theorem 3 proceeds along the lines of the corresponding theorem for $L_{\alpha}^{p}$ [2]. We shall see that with the help of Theorem 2 it is possible to omit the hypothesis $t F \subset F$, $0<t<1$, made in [2]. We need

LEMMA 8. Let $f \in \Lambda_{\alpha}^{p q}\left(R^{n}\right), 1<\alpha<\infty$. Then for $L_{\alpha}^{p q}$-a.e. $x, f$ is absolutely 
continuous on $H^{n-1}$-a.e. ray from $x$, and on such a ray fix $f(x+z)-f(x)=$ $\int_{0}^{1} \nabla f(x+t z) \cdot z d t$.

Proof. The proof is precisely the same as the proof of the corresponding lemma for $L_{\alpha}^{p}[2]$ as soon as we establish the existence of a sequence $\left\{f_{j}\right\} \subset C_{0}^{\infty}$ such that for $L_{\alpha}^{p q}$-a.e. $x$,

$$
G_{1} *\left|\nabla f_{j}-\nabla f\right|(x) \rightarrow 0 \text { as } j \rightarrow \infty \text {. }
$$

Let $0<\gamma<\min (1, \alpha-1)$, and choose $g \in \Lambda_{\gamma}^{p q}$ such that $f=G_{\alpha-\gamma} * g$. Let $\left\{f_{i}\right\} \subset C_{0}^{\infty}$ such that $f_{i} \rightarrow f\left(\Lambda_{\alpha}^{p q}\right)$, and let $g_{i} \in \Lambda_{\gamma}^{p q}$ with $f_{i}=G_{\alpha-\gamma} * g_{i}$. Then $g_{i} \rightarrow g\left(\Lambda_{\gamma}^{p q}\right)$. By Lemma $6, f_{k}(x) \rightarrow f(x)$ for $L_{\alpha}^{p q}$-a.e. $x$, for some subsequence $\left\{f_{k}\right\}$. Since $\alpha>1, \nabla f_{k} \rightarrow \nabla f\left(\Lambda_{\alpha-1}^{p q}\right)$, and hence $\nabla f_{k}-\nabla f=$ $G_{\alpha-\gamma-1} * h_{k}, h_{k} \rightarrow 0\left(\Lambda_{\gamma}^{p q}\right)$. Since $\gamma<1$, we easily see that $\left|h_{k}\right| \rightarrow 0\left(\Lambda_{\gamma}^{p q}\right)$, and hence for some subsequence $\left\{h_{i}\right\}, G_{\alpha-\gamma} *\left|h_{j}\right|(x) \rightarrow 0$ for $L_{\alpha}^{p q}$-a.e. $x$. Since $G_{1} * G_{\alpha-\gamma-1} *\left|h_{j}\right|(x)=G_{\alpha-\gamma} *\left|h_{j}\right|(x)$, the sequence $\left\{f_{j}\right\}$ has the desired property.

Proof of Theorem 3. Since $\alpha<1$ is Theorem 1, we may assume that $1<\alpha$. Let $f \in \Lambda_{\alpha}^{p q}$, and let $\Pi_{x}(y)=\Sigma_{0<|\beta|<k}\left(D^{\beta} f(x) / \beta !\right)(y-x)^{\beta}$; we observe that $D^{\beta} f \in \Lambda_{\alpha}^{p q}|\beta|^{\text {. }}$

Set $R_{x}(y)=f(y)-\Pi_{x}(y)$, and let $|\gamma|=k$. Then $D^{\gamma} R_{x}(y)=D^{\gamma} f(y)-$ $D^{\gamma} f(x)$. By Theorem 1 , for $L_{\alpha-k}^{p q}$ - a.e. $x$,

$$
\frac{1}{|S|} \int_{S+x}\left|D^{\gamma} R_{x}(y)\right| d y \rightarrow 0 \text { as } S \rightarrow 0,
$$

and by Theorem 2 ,

$$
\int_{0}^{1} \frac{1}{|t S|} \int_{t S+x}\left|D^{\gamma} R_{x}(y)\right| d y d t \rightarrow 0 .
$$

Let now $|\nu|=k-1$. For $L_{\alpha-k}^{p q}$-a.e. $x, D^{y} f$ is $\mathrm{AC}$ on $H^{n-1}$-a.e. ray from $x$, and on such a ray $D^{\nu} R_{x}(y)$ is also AC from which

$$
\left|D^{\nu} R(x+z)-D^{\nu} R_{x}(x)\right|<\int_{0}^{1}\left|\nabla\left(D^{\nu} R_{x}\right)(x+t z) \cdot z\right| d t .
$$

We note that $D^{\nu} R_{x}(x)=0$. As in [2] for any $S$ (not necessarily in $F$ )

(i) $\frac{1}{\delta(S)} \frac{1}{|S|} \int_{S+x}\left|D^{\nu} R_{x}(z)\right| d z \leqslant \int_{0}^{1} \frac{1}{|t S|} \int_{t S+x}\left|\nabla\left(D^{\nu} R_{x}\right)(z)\right| d z d t$.

If $S \in F$ and $S \rightarrow 0$, the integral goes to 0 for $L_{\alpha-k}^{p q}$-a.e. $x$.

For the next step of the proof we need

$$
\int_{0}^{1} \frac{1}{\delta(\tau S)} \frac{1}{|\tau S|} \int_{\tau S+x}\left|D^{\nu} R_{x}(z)\right| d z d \tau \rightarrow 0
$$

as $S \rightarrow 0, S \in F$, for $L_{\alpha-k}^{p q}$-a.e. $x$. From (i), replacing $S$ by $\tau S$, we obtain 


$$
\begin{aligned}
\int_{0}^{1} \frac{1}{\delta(\tau S)} & \frac{1}{|\tau S|} \int_{\tau S+x}\left|D^{\nu} R_{x}(z)\right| d z d \tau \\
& <\int_{0}^{1} \int_{0}^{1} \frac{1}{|t \tau S|} \int_{t \tau S+x}\left|\nabla\left(D^{\nu} R_{x}\right)(z)\right| d z d t d \tau
\end{aligned}
$$

and the corollary to Theorem 2 proves (ii).

If now $\eta$ is a mutli-index, $|\eta|=k-2$, we get as before

$$
\left|D^{\eta} R_{x}(x+z)\right| \leqslant \int_{0}^{1}\left|\nabla\left(D^{\eta} R_{x}\right)(x+t z) \cdot z\right| d t
$$

and hence for any $S$,

$$
\frac{1}{\delta(S)} \frac{1}{|S|} \int_{S+x}\left|D^{\eta} R_{x}(z)\right| d z \leqslant \int_{0}^{1} \frac{1}{|t S|} \int_{t S+x}\left|\nabla\left(D^{\eta} R_{x}\right)(z)\right| d z d t .
$$

If we divide this by $\delta(S) \geqslant \delta(t S)$, we finally obtain

$$
\begin{aligned}
& \frac{1}{\delta(S)^{2}} \frac{1}{|S|} \int_{S+x}\left|D^{\eta} R_{x}(z)\right| d z \\
& \quad<\int_{0}^{1} \frac{1}{\delta(t S)} \frac{1}{|t S|} \int_{t S+x}\left|\nabla\left(D^{\eta} R_{x}\right)(z)\right| d z .
\end{aligned}
$$

We apply now (ii).

For the step treating a multi-index of length $k-3$, we need to know that for $L_{\alpha-k}^{p q}$-a.e. $x$

$$
\int_{0}^{1} \frac{1}{\delta(\sigma S)^{2}} \frac{1}{|\sigma S|} \int_{\sigma S+x}\left|D^{\eta} R_{x}(z)\right| d z d \sigma \rightarrow 0
$$

as $S \rightarrow 0, S \in F$. This integral is majorized by

$$
\begin{aligned}
\int_{0}^{1} \int_{0}^{1} \frac{1}{\delta(t \sigma S)} \frac{1}{|t \sigma S|} \int_{t \sigma S+x}\left|\nabla\left(D^{\eta} R_{x}\right)(z)\right| d z d t d \sigma \\
<\sum \int_{0}^{1} \int_{0}^{1} \int_{0}^{1} \frac{1}{|\tau t \sigma S|} \int_{\tau t \sigma S+x}\left|\nabla\left(D^{\rho} R_{x}\right)(z)\right| d z d t d \sigma d \tau,
\end{aligned}
$$

where $\Sigma$ extends over all $\rho$ with $|\rho|=k-1$. By the corollary to Theorem 2 this is $o(1)$ as $S \rightarrow 0, S \in F$, for $L_{\alpha-k}^{p q}$-a.e. $x$. The proof of Theorem 3 is now complete.

6. We let $F$ be a family as in $\S 1$ and we shall assume now that

$$
\left\|M_{F} f\right\|_{p}<A_{p}\|f\|_{p}, \quad 1<p<\infty .
$$

The family of all oriented rectangles is an example satisfying (i). If (i) holds, then for a.e. $x$,

$$
\lim _{S \rightarrow 0} \frac{1}{|S|} \int_{S+x}|f(y)-f(x)|^{r} d y=0
$$


$f \in L^{p}$ and $1<r<p$. This is no longer true if $r=p$ as the example due to Saks [7] shows.

If $f \in \Lambda_{\alpha}^{p q}$, then $f \in L_{\beta}^{p}, \alpha>\beta$, and hence by Sobolov's theorem $f \in L^{r}$, where $1 / r=1 / p-\beta / n>1 / p-\alpha / n$. Here, of course, we assume that $\alpha p<n$. As in Theorem 1, we wish to study the size of the exceptional set (for which (ii) does not hold) under the hypothesis that $f \in \Lambda_{\alpha}^{p q}$. For this purpose we need a lemma.

Lemma 9. Let $f \in \Lambda_{\alpha}^{p q}, 0<\alpha<1$, and let $1 \leqslant r \leqslant p$. Then

$$
\left\|\mid f^{r}\right\|_{\Lambda_{a}^{p / r q}}<A_{r}\|f\|_{p}^{r-1}\|f\|_{\Lambda_{a}^{\rho q \sigma}}
$$

Proof. Recall that

$$
\|f\|_{\Lambda_{q}^{p q}}=\|f\|_{p}+\left\{\int_{R^{n}} \frac{\|f(x+t)-f(x)\|_{p}^{q}}{|t|^{n+\alpha q}} d t\right\}^{1 / q} .
$$

Now $|f(x+t)|^{r}-|f(x)|^{r}=(|f(x+t)|-|f(x)|) r \cdot \chi^{r-1}$, where $\chi$ is between $|f(x+t)|,|f(x)|$. Hence, if $\psi(x, t)=\max (|f(x+t)|,|f(x)|)$, we get

$$
\left.|| f(x+t)\right|^{r}-|f(x)|^{r}|\leqslant r| f(x+t)-f(x) \mid \psi(x, t)^{r-1} .
$$

For $\beta<p$ we have

$$
\left\||f(x+t)|^{r}-|f(x)|^{r}\right\|_{\beta} \leqslant r\left\{\int|f(x+t)-f(x)|^{\beta} \psi(x, t)^{\beta(r-1)} d x\right\}^{1 / \beta} .
$$

If we let $s=p / \beta, 1 / t=1-1 / s=(p-\beta) / p$, and apply Hölder's inequality, we get

$$
\begin{aligned}
& \left\|\left.f(x+t)\right|^{r}-|f(x)|^{r}\right\|_{\beta} \\
& \quad<r\left[\left\{\int|f(x+t)-f(x)|^{p} d x\right\}^{\beta / p}\left\{\int \psi(x, t)^{\beta(r-1) \cdot p /(p-\beta)}\right\}^{(p-\beta) / p}\right]^{1 / \beta} .
\end{aligned}
$$

We let now $\beta=p / r$. Then $\beta(r-1) p /(p-\beta)=p$, and $p \cdot(p-\beta) / p \beta=$ $r-1$, and hence

$$
\left\||f(x+t)|^{r}-|f(x)|^{r}\right\|_{p / r} \leqslant r\|f(x+t)-f(x)\|_{p}\|\psi(x, t)\|_{p}^{r-1} .
$$

Since $\psi(x, t)<|f(x)|+|f(x+t)|$, we see that $\|\psi(x, t)\|_{p}<2\|f\|_{p}$. Since, clearly, $\left\|\left.f\right|^{r}\right\|_{p / r}=\|f\|_{p}\|f\|_{p}^{r-1}$, the proof of the lemma is complete.

Let $C_{1}, C_{2}$ be two capacities on $R^{n}$, and let $\left[C_{1}, C_{2}\right](E)=\inf \left\{C_{1}\left(E^{\prime}\right)+\right.$ $\left.C_{2}\left(E^{\prime \prime}\right)\right\}$, where the inf is extended over all $E^{\prime}, E^{\prime \prime}$ with $E=E^{\prime} \cup E^{\prime \prime}$. It is easy to check that this is a capacity, and that $\left[C_{1}, C_{2}\right](E)<$ $\min \left(C_{1}(E), C_{2}(E)\right)$. If $\left\{C_{\alpha}\right\}$ is a collection of capacities on $R^{n}$, then $C(E)=$ $\sup _{\alpha} C_{\alpha}(E)$ is also a capacity on $R^{n}$.

Let $f \in \Lambda_{\alpha}^{p q}, \alpha p<n$. As we have seen, $f \in L^{r}$ for $1 / r>1 / p-\alpha / n$. If $p<r<s, 1 / s=1 / p-\alpha / n$, let for $r<t<s, \alpha_{t}=\alpha-n / p+n / t$. We 
define now the capacity $C_{r \alpha}$ by

$$
C_{r \alpha}(E)= \begin{cases}{\left[L_{\alpha}^{p q}, L_{\alpha}^{p / r q}\right](E),} & 1<r<p, \\ \sup _{t>r}\left[L_{\alpha_{t}}^{t q}, L_{\alpha}^{t / r q}\right](E), & p<r<s .\end{cases}
$$

THEOREM 4. Assume that $\left\|M_{F} f\right\|_{p} \leqslant A_{p}\|f\|_{p}, 1<p \leqslant \infty$. Let $f \in \Lambda_{\alpha}^{p q}$, $0<\alpha<1, \alpha p<n$, and let $1 / r>1 / p-\alpha / n$. Then for $C_{r \alpha}$-a.e. $x$,

$$
\frac{1}{|S|} \int_{S+x}|f(y)-f(x)|^{r} d y \rightarrow 0 \text { as } S \rightarrow 0 .
$$

Proof. We assume first that $1<r<p$. Let

$$
E=\left\{x: \limsup _{S \rightarrow 0} \frac{1}{|S|} \int_{S+x}|f(y)-f(x)|^{r} d y>0\right\},
$$

and let $0<\beta<\alpha$. By Lemma 3 it suffices to show that there exists a decomposition $E=E^{\prime} \cup E^{\prime \prime}$ such that $B_{\beta}^{p q}\left(E^{\prime}\right)=0$ and $B_{\beta}^{p / r q}\left(E^{\prime \prime}\right)=0$.

Let $\left\{\psi_{j}\right\} \subset C_{0}^{\infty}$ such that $\psi_{j} \rightarrow f\left(\Lambda_{\alpha}^{p q}\right)$. Then for each $j$

$$
\begin{aligned}
\left\{\frac{1}{|S|} \int_{S+x}|f(y)-f(x)|^{r} d y\right\}^{1 / r} \leqslant\left\{M_{F}\left(\left|f-\psi_{j}\right|^{r}\right)(x)\right\}^{1 / r} & \\
& +\left\{\frac{1}{|S|} \int_{S+x}\left|\psi_{j}(y)-\psi_{j}(x)\right|^{r} d y\right\}^{1 / r}+\left|\psi_{j}(x)-f(x)\right| \\
& =A_{1 j}(x)+A_{2 j}(x)+A_{3 j}(x) .
\end{aligned}
$$

We claim that there is $j_{1}<j_{2}<\cdots$ such that $M_{F}\left(\left|f-\psi_{j_{1}}\right|^{\prime}\right)(x) \rightarrow 0$ as $i \rightarrow \infty$ for $B_{\beta}^{p / r q}$-a.e. $x$.

By Lemma $9\left|f-\psi_{i}\right|^{r} \in \Lambda_{\alpha}^{p / r q}$, and hence $\left|f-\psi_{j}\right|^{r}=G_{\alpha-\beta} * g_{j}, g_{j} \in$ $\Lambda_{\beta}^{p / r q}$. Since

$$
\left\{x: A_{1 j}(x) \geqslant \varepsilon^{r}\right\} \subset\left\{x: G_{\alpha-\beta} * M_{F} g_{j}(x)>\varepsilon^{r}\right\},
$$

we obtain

$$
B_{\beta}^{p / r q}\left\{x: A_{1 j}(x)>\varepsilon^{r}\right\}<\frac{1}{\varepsilon^{r}}\left\|M_{F} g_{j}\right\|_{\Lambda_{\beta}^{p / q}}<\frac{A}{\varepsilon^{r}}\left\|g_{j}\right\|_{\Lambda_{\beta}^{p / q}} \quad \text { (Lemma 2). }
$$

By Lemma $9,\left|f-\psi_{j}\right|^{r} \rightarrow 0\left(\Lambda_{\alpha}^{p / r q}\right)$, and hence $g_{j} \rightarrow 0\left(\Lambda_{\beta}^{p / r q}\right)$. The claim now follows (see Lemma 6).

Let $E^{\prime \prime}=\left\{x: \lim \sup _{i \rightarrow \infty} M_{F}\left(\left|f-\psi_{j_{i}}\right|^{r}\right)(x)>0\right\}$. Then $B_{\beta}^{p / r q}\left(E^{\prime \prime}\right)=0$. We may assume that $\psi_{j_{i}}(x) \rightarrow f(x)$ for $L_{\alpha}^{p q}$-a.e. $x$, and if $E^{\prime}$ is the exceptional set, we have $B_{\beta}^{p q}\left(E^{\prime}\right)=0$. We show that $E \subset E^{\prime} \cup E^{\prime \prime}$.

Let $x \notin E^{\prime} \cup E^{\prime \prime}$, and let $\varepsilon>0$. Choose $j_{0}$ such that $A_{1 j_{0}}(x)<\varepsilon / 3, A_{3 j_{0}}(x)$ $<\varepsilon / 3$, and then choose $\tau_{0}$ such that $S \subset B\left(0, \tau_{0}\right)$ implies $A_{2 j_{0}}(x)<\varepsilon / 3$. It follows then that $x \notin E$.

If $p<r<s, 1 / s=1 / p-\alpha / n$, let $r<t<s$, and let $\alpha_{t}=\alpha-n / p+$ 
$n / t$. By [6, p. 441], $f \in \Lambda_{\alpha_{1}}^{t q}$. Consequently by the first part of the proof, $\left[L_{\alpha_{1}}^{t q}, L_{\alpha_{1}}^{t / r q}\right](E)=0$, and the proof of the theorem is complete.

\section{REFERENCES}

1. A. P. Calderon and A. Zygmund, Local properties of solutions of elliptic partial differential equations, Studia Math. 20 (1961), 171-225. MR 25 \#310.

2. D. J. Deignan and W. P. Ziemer, Strong differential properties of Bessel potentials, Trans. Amer. Math. Soc. 225 (1977), 113-122.

3. M. deGuzmán, Differentiation of integrals in $R^{n}$, Lecture Notes in Math., no. 481, SpringerVerlag, Berlin and New York, 1975.

4. N. Meyers, A theory of capacities for potentials of functions in Lebesgue classes, Math. Scand. 26 (1970), 255-292. MR 43 \#3474.

5. E. M. Stein, Singular integrals and differentiability properties of functions, Princeton Univ. Press, Princeton, N. J., 1970. MR 44 \# 7280.

6. M. H. Taibleson, On the theory of Lipschitz spaces of distributions on Euclidean n-space, J. Math. Mech. 13 (1964), 407-479. MR 29 \#462.

7. S. Saks, Remark on the differentiability of the Lebesgue indefinite integral, Fund. Math. 22 (1934), 257-261.

8. N. Aronszajn and K. T. Smith, Theory of Bessel potentials. I, Ann. Inst. Fourier 11 (1961), 385-475. MR 26 \# 1485.

Mathematical Sciences Division, Purdue University, West Lafayette, Indiana 47907 